\title{
A New Multidisciplinary Design Optimization Method Accounting for Discrete and Continuous Variables under Aleatory and Epistemic Uncertainties
}

\author{
Hong-Zhong Huang \\ School of Mechatronics Engineering, University of Electronic Science and Technology of China, No. 2006, Xiyuan \\ Avenue, West Hi-Tech Zone, Chengdu, Sichuan, 611731, P.R. China \\ E-mail: hzhuang@uestc.edu.cn \\ Xudong Zhang \\ School of Mechatronics Engineering, University of Electronic Science and Technology of China, No. 2006, Xiyuan \\ Avenue, West Hi-Tech Zone, Chengdu, Sichuan, 611731, P.R. China \\ De-Biao Meng \\ School of Mechatronics Engineering, University of Electronic Science and Technology of China, No. 2006, Xiyuan \\ Avenue, West Hi-Tech Zone, Chengdu, Sichuan, 611731, P.R. China \\ Yu Liu \\ School of Mechatronics Engineering, University of Electronic Science and Technology of China, No. 2006, Xiyuan \\ Avenue, West Hi-Tech Zone, Chengdu, Sichuan, 611731, P.R. China \\ Yan-Feng Li \\ School of Mechatronics Engineering, University of Electronic Science and Technology of China, No. 2006, Xiyuan \\ Avenue, West Hi-Tech Zone, Chengdu, Sichuan, 611731, P.R. China \\ Received 6 October 2010 \\ Accepted 22 November 2011
}

\begin{abstract}
Various uncertainties are inevitable in complex engineered systems and must be carefully treated in design activities. Reliability-Based Multidisciplinary Design Optimization (RBMDO) has been receiving increasing attention in the past decades to facilitate designing fully coupled systems but also achieving a desired reliability considering uncertainty. In this paper, a new formulation of multidisciplinary design optimization, namely RFCDV (random/fuzzy/continuous/discrete variables) Multidisciplinary Design Optimization (RFCDV-MDO), is developed within the framework of Sequential Optimization and Reliability Assessment (SORA) to deal with multidisciplinary design problems in which both aleatory and epistemic uncertainties are present. In addition, a hybrid discrete-continuous algorithm is put forth to efficiently solve problems where both discrete and continuous design variables exist. The effectiveness and computational efficiency of the proposed method are demonstrated via a mathematical problem and a pressure vessel design problem.
\end{abstract}

Keywords: Multidisciplinary design optimization (MDO), Aleatory uncertainty, Epistemic uncertainty, Continuous/discrete variables, Random/Fuzzy/Continuous/Discrete Variables Multidisciplinary Design Optimization (RFCDV-MDO), Sequential Optimization and Reliability Assessment (SORA). 


\section{Introduction}

In the last two decades, uncertainty has been a focus of engineering design for complex and coupled systems [16]. In practical engineering design problems such as aircraft and ship design, the process is coupled and specialized design groups are involved. These specialists usually have engineering responsibility for particular design disciplines. Design decisions made by one group are either impacted by or impact on the decisions made by other groups. In some cases, different groups generate conflicting design proposals. In order to preserve the coupling that naturally exists among the groups of the whole engineering team, the optimization model must include a degree of coordination. Multidisciplinary Design Optimization (MDO) is a body of methods and techniques for performing such optimization so as to balance the design considerations. Moreover, uncertainties in MDO have a significant influence on the whole design process because uncertainties may propagate through linking variables and the effect of uncertainties could accumulate. If uncertainties are not considered, optimization results may be unreliable.

Reliability Based Multidisciplinary Design Optimization (RBMDO) has been widely applied for the the requirements of high reliability or safety in complex and coupled systems [7-14]. Response surface models created at the system level are employed to replace the computationally expensive simulation models in order to release the computational requirements of reliability analysis involved in MDO under uncertainty [7]. The framework for RBMDO is proposed in [8] wherein reliability analysis is decoupled from the optimization. Reliability is initially computed before the first execution of the optimization loop. Then updated iteratively after each optimization loop during which approximate forms of reliability constraints are used. A multi-stage, parallel implementation of probabilistic design optimization is utilized with the aim of integrating the existing reliability analysis methods into the MDO framework in [9]. Concurrent subsystem optimizations were proposed in [10-12] and collaborative reliability analysis methods were used [13] to search for the Most Probable Point (MPP). Sequential Optimization and Reliability Assessment (SORA) method for RBMDO was proposed in [14]. From design optimization, SORA is based on the idea of decoupling reliability analysis. Using the MPP obtained from the previous iteration, the constraint in deterministic optimization is modified to make sure that the MPP of the current iteration falls into the feasible region. A new design point is obtained and followed by reliability assessment to check the feasibility of each reliability constraint at the new design point after solving the deterministic optimization. Generally, the whole process will converge in a few iterations.

However, most existing RBMDO methods are only able to deal with uncertainty based on the probability theory. In many practical engineering designs, both Aleatory Uncertainty (AU) and Epistemic Uncertainty (EU) are associated with design inputs. AU include stochastic uncertainty, irreducible uncertainty, inherent uncertainty, and variability. The design variables with AU can be treated as random variables. They may be modeled with probability theory. EU represents reducible uncertainty and subjective uncertainty. EU caused by lack of knowledge can be modeled with possibility theory. The design variables with EU can be treated as fuzzy variables [15-16]. The challenge is how to efficiently propagate the effect of $\mathrm{AU}$ and $\mathrm{EU}$ respectively in the context of multidisciplinary analysis and design. Up to now, when both AU and EU exist in inputs, inputs with EU are characterized as random variables in MDO, and their distributions are inferred from the limited data, and then RBMDO is carried out to find the optimum design. It has been pointed out in [17], in singular disciplinary, the optimum design obtained with the above method may be unbelievable and unsafe. Results may be even worse because of coupling of multiple disciplines. In [18], both types of uncertainty are considered in singular discipline design based on the idea of conditional possibility of failure. A method of Maximal Failure Search (MPS) was proposed. As a result, the design problems become more complicated in MDO. Few works have been done in this situation. Furthermore, both discrete and continuous variables may co-exist in practical engineering design. So far, almost all existing works focus on MDO assuming continuous variables. As not only continuous variables but also discrete variables should be considered in practical engineering design, methods need to be developed to take these features into account.

This paper proposes a formulation of RFCDVMDO, a method of RFCDV-MDO in the framework of SORA called RFCDV-MDO-SORA, and a hybrid 
discrete-continuous algorithm to deal with discretecontinuous optimization problems. This method has the advantage that $\mathrm{AU}$ and $\mathrm{EU}$ can be measured using probability theory and possibility theory, respectively. Meanwhile discrete and continuous variables can be considered in MDO problems. A mathematical example and a pressure vessel design problem are used to demonstrate the application of the proposed method. The advantages of the proposed method are compared to the RBMDO-SORA.

The reminder of this paper is organized as follows. In Section 2, fundamental analysis of reported probability/possibility models is given. In Section 3, the formulation of RFCDV (random/fuzzy/continuous/ discrete variables) Multidisciplinary Design Optimization (RFCDV-MDO) is provided. In Section 4, the procedure of the RFCDV-MDO-SORA is interpreted in detail, subsequently the formulation mentioned in this method is proposed. The algorithm is proposed in Section 5. A mathematical example and a pressure vessel design problem are used to verify the effectiveness of the RFCDV-MDO-SORA in Section 6, followed by conclusions in Section 7 .

\section{Fundamental analysis}

$\mathrm{AU}$ and $\mathrm{EU}$ are treated as random and fuzzy variables, respectively. In [18], the case in which all random and fuzzy variables are continuous is discussed in a single discipline. Suppose that the random variables $\mathbf{X}_{r}$ are subjected to the joint probability density function $f_{\mathbf{X}_{r}}\left(\mathbf{X}_{r}\right)$; the fuzzy variables $\mathbf{X}_{f}$ have the membership function $\Pi_{\mathbf{X}_{f}}\left(\mathbf{X}_{f}\right)$ and the failure mode is $G\left(\mathbf{x}_{r}, \mathbf{x}_{f}\right)>0$.

The possibility of failure is computed as follows. Firstly we assume that the fuzzy variables are fixed at $\mathbf{X}_{f}=\mathbf{x}_{f}$. The conditional probability of failure is evaluated as $P_{f} \mid\left\{\mathbf{X}_{f}=\mathbf{x}_{f}\right\}=\int_{G\left(\mathbf{x}_{r}, \mathbf{x}_{f}\right)>0} f_{\mathbf{X}_{r}}\left(\mathbf{x}_{r}\right) d \mathbf{x}_{r}$. The conditional possibility of failure is set to be equal to this conditional probability of failure. At last, the possibility of failure is computed by

$$
\begin{aligned}
\Pi_{f} & =\sup _{\mathbf{x}_{f}}\left[\min \left\{\Pi_{f} \mid\left(\mathbf{X}_{f}=\mathbf{x}_{f}\right), \Pi_{\mathbf{x}_{f}}\left(\mathbf{x}_{f}\right)\right\}\right] \\
& =\sup _{\mathbf{x}_{f}}\left[\min \left\{P_{f} \mid\left(\mathbf{X}_{f}=\mathbf{x}_{f}\right), \Pi_{\mathbf{x}_{f}}\left(\mathbf{x}_{f}\right)\right\}\right] \\
& =\sup _{\mathbf{x}_{f}}\left[\min \left\{\int_{\mathbf{x}_{r}: G\left(\mathbf{x}_{r}, \mathbf{x}_{f}\right)>0} f_{\mathbf{X}_{r}}\left(\mathbf{x}_{r}\right) d \mathbf{x}_{r}, \Pi_{\mathbf{x}_{f}}\left(\mathbf{x}_{f}\right)\right\}\right]
\end{aligned}
$$

We have also assumed that all random variables and random parameters are mutually independent, and all fuzzy variables and fuzzy parameters are noninteractive.

The fuzzy discrete variable is the fuzzy variable which can only take a series of integers or some special values. For example, if $X_{f d i}=\frac{0.6}{4}+\frac{0.8}{5}+\frac{1}{6}+\frac{0.8}{7}+\frac{0.6}{8}$ and the failure value is set as 0.0001 , in possibility analysis, $X_{f d i}$ can only take one of $(4,5,6,7,8)$.

In the following formulations, $\mathbf{x}_{f}$ denotes $\left(\mathbf{x}_{f c}, \mathbf{x}_{f d}\right)$ and $\mathbf{p}_{f}$ indicates $\left(\mathbf{p}_{f c}, \mathbf{p}_{f d}\right)$. The possibility of failure can be calculated by the following steps.

First, fix the fuzzy variables and parameters at $\mathbf{x}_{f}=\left(\mathbf{x}_{f c}, \mathbf{x}_{f d}\right), \mathbf{p}_{f}=\left(\mathbf{p}_{f c}, \mathbf{p}_{f d}\right) \quad, \quad$ the conditional probability of failure can be calculated by $P_{f} \mid\left\{\mathbf{X}_{f}=\mathbf{x}_{f}, \mathbf{P}_{f}=\mathbf{p}_{f}\right\}$

$$
=\sum_{k=1}^{N}\left\{\begin{array}{l}
\int_{\mathbf{x}_{r c}, \mathbf{p}_{r c}: G\left(\begin{array}{l}
\mathbf{X}_{r,}, \mathbf{k}_{r d}^{k}, \mathbf{x}_{f}, \\
\mathbf{p}_{r r}, \mathbf{p}_{r d}^{k}, \mathbf{p}_{f}
\end{array}\right)>0} f_{\mathbf{X}_{r c}\left(\mathbf{x}_{r c}\right) f_{\mathbf{P}_{r c}}\left(\mathbf{p}_{r c}\right) d \mathbf{x}_{r c} d \mathbf{p}_{r c}} \\
\times F_{\mathbf{X}_{r d}}\left(\mathbf{x}_{r d}^{k}\right) \times F_{\mathbf{P}_{r d}}^{k}\left(\mathbf{p}_{r d}^{k}\right)
\end{array}\right\}
$$

where $N$ stands for the number of all possible combinations of $\mathbf{x}_{r d}$ and $\mathbf{p}_{r d}$.

Second, set the possibility of failure to be the same as the calculated probability of failure. Note that possibility is an alterative measure when the actual probability is difficult to compute. So if there exists the probability, one can set the possibility to be the same as the probability [18]. 


$$
\begin{aligned}
& \Pi_{f}=\sup _{\mathbf{x}_{f}, \mathbf{p}_{f}}\left[\min \left\{\Pi_{f} \mid\left(\mathbf{X}_{f}=\mathbf{x}_{f}, \mathbf{P}_{f}=\mathbf{p}_{f}\right), \Pi_{\mathbf{x}_{f}, \mathbf{p}_{f}}\left(\mathbf{x}_{f}, \mathbf{p}_{f}\right)\right\}\right] \\
& =\sup _{\mathbf{x}_{f}, \mathbf{p}_{f}}\left[\min \left\{P_{f} \mid\left(\mathbf{X}_{f}=\mathbf{x}_{f}, \mathbf{P}_{f}=\mathbf{p}_{f}\right), \Pi_{\mathbf{x}_{f}, \mathbf{p}_{f}}\left(\mathbf{x}_{f}, \mathbf{p}_{f}\right)\right\}\right]
\end{aligned}
$$

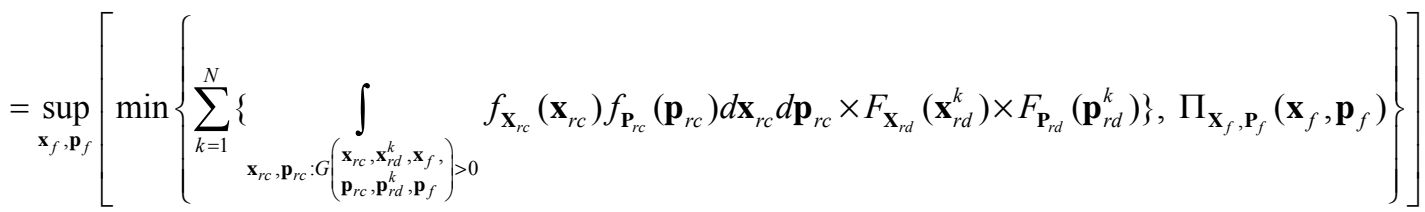

It is impractical to calculate the sum in Eq. (1) using the analytical method directly. To resolve this difficulty, Eq. (1) can be written as:

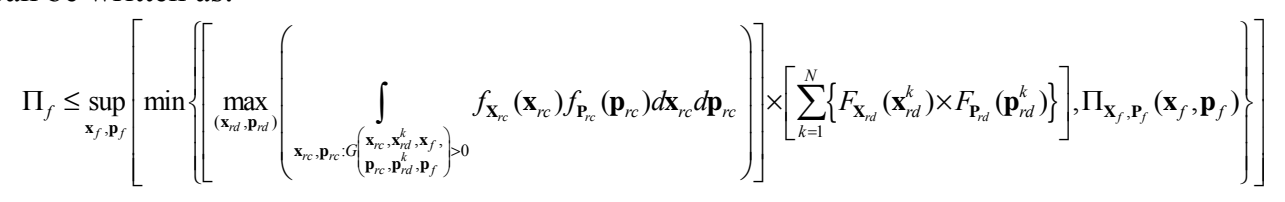

where $N$ stands for the number of all possible combinations of $\mathbf{x}_{r d}$ and $\mathbf{p}_{r d}$.

The value of $\sum_{k=1}^{N}\left\{F_{\mathbf{X}_{r d}}\left(\mathbf{x}_{r d}^{k}\right) \times F_{\mathbf{P}_{r d}}\left(\mathbf{p}_{r d}^{k}\right)\right\}$ can be obtained initially in design. It is the sum of probabilities of all combinations of discrete random variables and parameters.

To facilitate the calculation, all continuous random variables and random parameters should be transformed into the standard normal variables and parameters in Uspace using Rosenblatt transformation [14], and all the fuzzy variables and fuzzy parameters (discrete and continuous) should be translated into the standard fuzzy ones in V-space [18]. The standard fuzzy variable has the isosceles triangular membership function as:

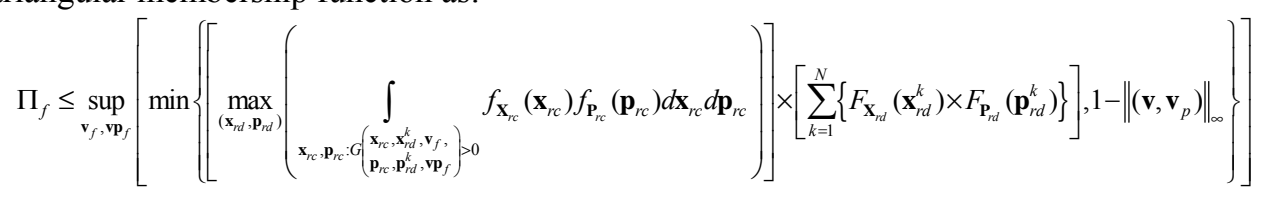

If the right hand side of Eq. (4) is not larger than $\alpha_{t}$, then the possibility of failure $\Pi_{f} \leq \alpha_{t}$.

When given the design point at $\mathbf{X}^{M}$, there are three cases among all points satisfying the condition

(ii) $1-\left\|\left(\mathbf{v}, \mathbf{v}_{p}\right)\right\|_{\infty}=\alpha_{t}$;

(iii) $1-\left\|\left(\mathbf{v}, \mathbf{v}_{p}\right)\right\|_{\infty}>\alpha_{t}$.

If the fuzzy part satisfies case (i) or case (ii), $G(\mathbf{X}, \mathbf{P})>0$ :

(i) $1-\left\|\left(\mathbf{v}, \mathbf{v}_{p}\right)\right\|_{\infty}<\alpha_{t}$;

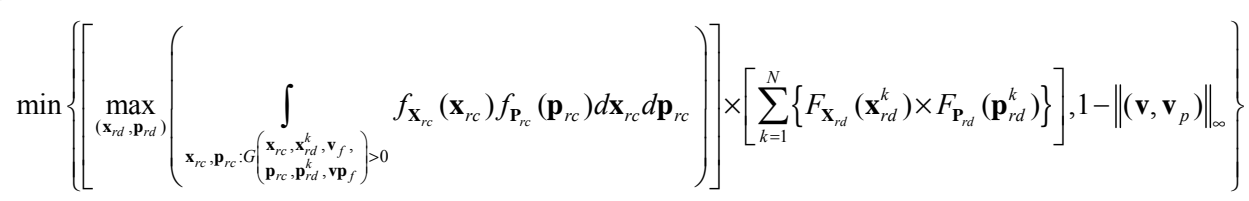


Then, cases 1 and 2 do not affect the final result of

$$
\Pi_{f} \leq \alpha_{t} \text {.If }
$$

$$
\left[\max _{\left(\mathbf{x}_{r d}, \mathbf{p}_{r d}\right)}\left(\int_{\mathbf{x}_{r c}, \mathbf{p}_{r c}: G\left(\begin{array}{l}
\mathbf{x}_{r c}, \mathbf{x}_{r d}^{k}, \mathbf{x}_{f}, \\
\mathbf{p}_{r c}, \mathbf{p}_{r d}^{k}, \mathbf{p}_{f}
\end{array}\right)>0} f_{\mathbf{X}_{r c}}\left(\mathbf{x}_{r c}\right) f_{\mathbf{P}_{r c}}\left(\mathbf{p}_{r c}\right) d \mathbf{x}_{r c} d \mathbf{p}_{r c}\right)\right] \times\left[\sum_{k=1}^{N}\left\{F_{\mathbf{X}_{r d}}\left(\mathbf{x}_{r d}^{k}\right) \times F_{\mathbf{p}_{r d}}\left(\mathbf{p}_{r d}^{k}\right)\right\}\right] \leq \alpha_{t}
$$

whenever the fuzzy part satisfies case (3), then the possibility of failure $\Pi_{f} \leq \alpha_{t}$.

There is a similar formulation in the method of Performance Measure Approach (PMA) to check whether or not $\Pi_{f} \leq \alpha_{t}$ at the design point as follows:

$$
\begin{array}{ll}
\max & G\left(\mathbf{X}_{d}, \mathbf{U}_{\mathbf{X} c}, \mathbf{V}_{\mathbf{X}}, \mathbf{P}_{d}, \mathbf{U}_{\mathbf{P}_{c}}, \mathbf{V}_{\mathbf{P}}\right) \\
\text { s.t. } & \left\|\left(\mathbf{U}_{\mathbf{X} c}, \mathbf{U}_{\mathbf{P} c}\right)\right\|_{2} \leq-\Phi^{-1}\left[\frac{\alpha_{t}}{\sum_{k=1}^{N}\left\{F_{\mathbf{X}_{r d}}\left(\mathbf{x}_{r d}^{k}\right) \times F_{\mathbf{P}_{r d}}\left(\mathbf{P}_{r d}^{k}\right)\right\}}\right] \\
& \left\|\left(\mathbf{V}_{\mathbf{X}}, \mathbf{V}_{\mathbf{P}}\right)\right\|_{\infty}<1-\alpha_{t}
\end{array}
$$

where $\mathbf{X}_{r d}, \mathbf{P}_{r d}$ vary in all allowable combined modes.

The solution is denoted by $\mathbf{X}_{d}^{*}, \mathbf{U}_{\mathbf{X} c}^{*}, \mathbf{V}_{\mathbf{X}}^{*}, \mathbf{P}_{d}^{*}, \mathbf{U}_{\mathbf{P} c}^{*}, \mathbf{V}_{\mathbf{P}}^{*}$.

$G\left(\mathbf{X}_{d}^{*}, \mathbf{U}_{\mathbf{X} c}^{*}, \mathbf{V}_{\mathbf{X}}^{*}, \mathbf{P}_{d}^{*}, \mathbf{U}_{\mathbf{P} c}^{*}, \mathbf{V}_{\mathbf{P}}^{*}\right)$ is the value of the performance measure at the MPPP. If the maximal value is not larger than zero, the current design point is feasible, otherwise infeasible.

The above formulation is given for the singular discipline. When performing uncertainty analysis in the environment of MDO, there are extra computations for the linking variables to achieve consistency between multiple disciplines. In [14], two methods are adopted: one is that the consistency is maintained by using extra constraints; the other is that the values of the linking variables are obtained by solving an optimization problem of consistency.

Although either method may be used, the number of disciplinary analyses in the former is less than that of the latter and the former is more stable than the latter [14]. So in this paper, the first method is adopted, i.e. consistency is treated as extra constraints.

In our fundamental analysis in this section, the formulation of uncertainty analysis (probability/possibility analysis) in the environment of MDO is not provided, but it will be provided in Section 4.

\section{RFCDV-MDO}

RFCDV-MDO deals with the uncertaint continuous/discrete design variables or parameters as quantities that can be measured with probability theory or possibility theory respectively. The formulation of RFCDV-MDO is

$$
\begin{array}{cl}
\min _{\left(\mathbf{d}_{s}, \mathbf{d}, \mathbf{X}_{s}^{M}, \mathbf{X}^{M}\right)} & f\left(\mathbf{d}_{s}, \mathbf{d}, \mathbf{X}_{s}^{M}, \mathbf{X}^{M}, \mathbf{P}^{M}, \mathbf{Y}^{M}\right) \\
\text { s.t. } \quad & \Pi\left[G^{(i)}\left(\mathbf{d}_{s}, \mathbf{d}_{i}, \mathbf{X}_{s}, \mathbf{X}_{i}, \mathbf{P}_{i}, \mathbf{Y}_{\bullet i}\right)>0\right] \leq \alpha_{t} \\
& g^{(i)}\left(\mathbf{d}_{s}, \mathbf{d}_{i}, \mathbf{X}_{s}^{M}, \mathbf{X}_{i}^{(i), M}, \mathbf{P}_{i}^{M}, \mathbf{Y}_{\bullet i}^{M}\right) \leq 0 \\
& \mathbf{d}_{s}^{L} \leq \mathbf{d}_{s} \leq \mathbf{d}_{s}^{U}, \mathbf{d}^{L} \leq \mathbf{d} \leq \mathbf{d}^{U}, \\
& \mathbf{X}_{s}^{M, L} \leq \mathbf{X}_{s}^{M} \leq \mathbf{X}_{s}^{M, U}, \mathbf{X}^{M, L} \leq \mathbf{X}^{M} \leq \mathbf{X}^{M, U}, \\
& i=1,2, \cdots, n d
\end{array}
$$

\section{RFCDV-MDO in the framework of SORA (RFCDV-MDO- SORA)}

Sequential Optimization and Reliability Assessment (SORA) is developed for Reliability Based Design Optimization (RBDO) originally and is introduced into MDO in [14]. SORA is further developed to deal with MDO with various kinds of variables having different uncertainties, named RFCDV-MDO-SORA utilizing the idea of SORA. The method is explained in details including strategy, procedure and the formulations.

\subsection{Strategy of RFCDV-MDO -SORA}

To solve the RFCDV-MDO problem efficiently, we adopt the following two technologies:

(i) Performance Measure Approach (PMA). PMA is a method that is more efficient than evaluating the actual probability or possibility $[14,17,18]$. Some non-active probability or possibility constraints may dominate the whole computational process when directly evaluating their actual probability or possibility. This will decrease the computational efficiency. However, if we utilize PMA, the probability or possibility of failure is initially set to an acceptable value and treated as constraints. The maximum value of the probability or possibility constraint function (performance measure) is 
calculated. In this paper, the failure mode is defined as $G(\cdot)>0$. The probability or possibility requirement is met when the value is not larger than zero.

(ii) Sequential Optimization and Reliability Assessment (SORA). The solution process of MDO under uncertainties is decoupled into the solution of MDO and the solution of probability/possibility analysis with the idea of SORA [14]. Probability/possibility analysis is executed after a deterministic MDO in each iteration. The mean value or maximum grade point of each design variable is obtained after solving the deterministic MDO. To analyze the feasibility of each probability/possibility constraint at the optimum and to obtain the MPPP and the value of performance measure of each probability/ possibility constraint probability/ possibility analysis is applied. If some of the probability/possibility constraints performance measures are larger than zero, the MPPPs relevant to all constraints are used to reconstruct a deterministic MDO for the next iteration to improve the feasible design. Based on this, the MDO problem and the uncertainty analysis are not nested but sequential. So the efficiency is improved and it is expected that the whole process will converge in a few iterations.

\subsection{Procedure of Establishment}

In this section, the procedure of RFCDV-MDO-SORA is illustrated step by step.

Step 1: Set the initial value for

$\mathbf{d}_{s}^{(0)}, \mathbf{d}^{(0)}, \mathbf{X}_{s}^{M,(0)}, \mathbf{X}^{M,(0)} ; k=1$

Step 2: Solve the deterministic MDO. To obtain the values of $\mathbf{d}_{s}^{(k)}, \mathbf{d}^{(k)}, \mathbf{X}_{s}^{M,(k)}, \mathbf{X}^{M,(k)}$ is the aim of solving the deterministic MDO. Because there is no information about the MPPPs in the first iteration, the MPPPs are set to be equivalent to $\mathbf{X}_{s}^{M,(0)}, \mathbf{X}^{M,(0)}, \mathbf{P}^{M}$. Deterministic variables, the mean value or maximum grade point of each random or fuzzy continuous or discrete variable are the variables in the deterministic constraints.

However, constraints in the deterministic MDO are modified with the MPPPs obtained in the previous iteration when the requirements of probability/possibility constraints are not all satisfied from the second iteration.
Step 3: Probability/possibility analysis. First, the continuous random variables and the continuous parameters are transformed into standard normal ones in U-space using Rosenblatt transformation; meanwhile all fuzzy variables and fuzzy parameters whether continuous or discrete are transformed into standard fuzzy ones in V-space using Eq. (3). Then to check the feasibility of each probability/possibility constraint at the design point obtained in Step 2 , probability/possibility analysis is carried out. And the results are MPPP and performance measure at MPPP corresponding to each probability/possibility constraint.

Step 4: Check convergence. If the requirements of probability/possibility constraints are all satisfied and the value of the objective is stable $\left(G_{i} \leq 0, i=1 \sim n d ;|f(k)-f(k-1)| \leq \varepsilon\right)$, where $\varepsilon$ is an arbitrary small positive constant, stop the process of solution; otherwise set $k=k+1$ and go to Step 2 with the MPPPs obtained in Step 3.

If the requirement of the probability/possibility constraint $G^{(i)}$ is not satisfied $\left(G^{(i)}>0\right)$, then the MPPP $\mathbf{X}_{s}^{*,(i),(k-1)}, \mathbf{X}^{*,(i),(k-1)}, \mathbf{P}^{*,(i),(k-1)}$ obtained from the probability/possibility analysis in iteration $k-1$ will be used to modify the constraint in the $k$ th deterministic MDO. To ensure the feasibility of the probability/possibility constraint, the $k$ th MPPP should fall into the deterministic feasible region. Let $\mathbf{S}$ be the shift vector. The deterministic constraint in the $k$ th MDO is modified as: $G^{(i)}\left(\mathbf{X}^{M}-\mathbf{S}\right) \leq 0$.

In this paper, two methods of using shift vectors are used. The first method is based on the idea of the SORA in [14] as:

$$
\begin{aligned}
& \mathbf{S}=\left\{\mathbf{S}_{c}^{(i)}, \mathbf{S}_{d}^{(i)}\right\} \\
& \mathbf{S}_{c}^{(i), k}=\mathbf{X}_{c}^{M,(k-1)}-\mathbf{X}_{c}^{*,(i),(k-1)} \\
& \mathbf{S}_{d}^{(i), k}=\mathbf{X}_{d}^{M,(k-1)}-\mathbf{X}_{d}^{*,(i),(k-1)}
\end{aligned}
$$

where $\mathbf{S}_{c}$ indicates the shifts of variables with uncertainty, whose mean value or maximum grade point is continuous; while $\mathbf{S}_{d}$ indicates the shifts of the variables with uncertainty, whose mean value or maximal grade point is discrete.

The second shift vector is constructed as: 


$$
\begin{aligned}
& \mathbf{S}=\left\{\mathbf{S}_{c}^{(i)}, \mathbf{S}_{d}^{(i)}\right\} \\
& \mathbf{S}_{c}^{(i), k}=\mathbf{X}_{c}^{M,(k-1)}-\mathbf{X}_{c}^{*,(i),(k-1)} \\
& \mathbf{S}_{d}^{(i), k}= \begin{cases}\sum_{h=1}^{j} \Delta_{i h} & \text { if }\left(\mathbf{X}_{d}^{M,(k-1)}-\mathbf{X}_{d}^{*,(i),(k-1)}\right)>0 \text { and } \sum_{h=1}^{j-1} \Delta_{i h}<\left(\mathbf{X}_{d}^{M,(k-1)}-\mathbf{X}_{d}^{*,(i),(k-1)}\right) \leq \sum_{h=1}^{j} \Delta_{i h} \\
-\sum_{h=1}^{j} \Delta_{i h} & \text { if }\left(\mathbf{X}_{d}^{M,(k-1)}-\mathbf{X}_{d}^{*,(i),(k-1)}\right)<0 \text { and }-\sum_{h=1}^{j} \Delta_{i h} \leq\left(\mathbf{X}_{d}^{M,(k-1)}-\mathbf{X}_{d}^{*,(i),(k-1)}\right)<-\sum_{h=1}^{j-1} \Delta_{i h}\end{cases}
\end{aligned}
$$

where $\left(\mathbf{X}_{d}^{M,(k-1)}-\mathbf{X}_{d}^{*,(k-1)}\right)^{i}$ indicates the $i$ th component of $\mathbf{X}_{d}^{M}-\mathbf{X}_{d}^{*}, \Delta_{i 1}, \Delta_{i 2}, \cdots, \Delta_{i j}, \cdots, \Delta_{i n}$ are the discrete increments from $\mathbf{X}_{d}^{M,(k-1)}$ when the value of $\left(\mathbf{X}_{d}^{M,(k-1)}-\mathbf{X}_{d}^{*,(k-1)}\right)^{i}$ is larger than zero, $\Delta_{i 1}, \Delta_{i 2}, \cdots, \Delta_{i j}, \cdots, \Delta_{i n}$ are the discrete decrements from $\mathbf{X}_{d}^{M,(k-1)}$ when the value of $\left(\mathbf{X}_{d}^{M,(k-1)}-\mathbf{X}_{d}^{*,(k-1)}\right)^{i}$ is less than zero. The values of the MPPPs $\mathbf{P}_{i}^{*,(i),(k-1)}$ directly substitute $\mathbf{P}_{i}^{(i)}$ in the probability/possibility constraint. The probability/possibility constraint is modified into:

$$
G_{\Pi}^{(i)}\left(\mathbf{d}_{s}, \mathbf{d}_{i}, \mathbf{X}_{s}^{M}-\mathbf{S}_{s}, \mathbf{X}_{i}^{M}-\mathbf{S}_{i}, \mathbf{P}_{i}^{*,(i),(k-1)}, \mathbf{Y}_{\bullet}^{(i)}\right) \leq 0 .
$$

Fig. 1 shows the flowchart of the RFCDV-MDOSORA approach. The following are the formulations for the deterministic MDO and the probability/possibility analysis.

\subsection{Formulations for deterministic RFCDV- MDO and probability/possibility analysis}

The deterministic RFCDV-MDO model of the $k$ th iteration can be expressed as follows.

$$
\begin{array}{ll}
\min _{\left(\mathbf{d}_{s}, \mathbf{d}, \mathbf{X}_{s}^{M}, \mathbf{X}^{M}\right)} & f\left(\mathbf{d}_{s}, \mathbf{d}, \mathbf{X}_{s}^{M}, \mathbf{X}^{M}, \mathbf{P}^{M}, \mathbf{Y}^{M}\right) \\
\text { s.t. } \quad & G_{\Pi}^{(i)}\left(\mathbf{d}_{s}, \mathbf{d}_{i}, \mathbf{X}_{s}^{*,(i),(k-1)}, \mathbf{X}_{i}^{*,(i),(k-1)}, \mathbf{P}_{i}^{*,(i),(k-1)}, \mathbf{Y}_{\bullet}^{*,(i)}\right) \leq 0 \\
& g^{(i)}\left(\mathbf{d}_{s}, \mathbf{d}_{i}, \mathbf{X}_{s}^{M}, \mathbf{X}_{i}^{(i), M}, \mathbf{P}_{i}^{M}, \mathbf{Y}_{\bullet_{i}}^{M}\right) \leq 0 \\
& i=1,2, \cdots, n d \\
y_{i j}^{M}=y_{i j}\left(\mathbf{d}_{s}, \mathbf{d}_{i}, \mathbf{X}_{s}^{M}, \mathbf{X}_{i}^{(i), M}, \mathbf{P}_{i}^{M}, \mathbf{Y}_{\bullet i}^{M}\right) \\
i, j=1,2, \cdots, n d, i \neq j \\
y_{j m}^{*,(i)}=y_{j m}^{*,(i)}\left(\mathbf{d}_{s}, \mathbf{d}_{i}, \mathbf{X}_{s}^{*,(i),(k-1)}, \mathbf{X}_{j}^{*,(i),(k-1)}, \mathbf{P}_{j}^{*,(i),(k-1)}, \mathbf{Y}_{\bullet_{j}^{*}}^{*,(i)}\right) \\
i, j, m=1,2, \cdots, n d, j \neq m \\
\mathbf{d}_{s}^{L} \leq \mathbf{d}_{s} \leq \mathbf{d}_{s}^{U}, \mathbf{d}^{L} \leq \mathbf{d} \leq \mathbf{d}^{U}, \\
\mathbf{X}_{s}^{M, L} \leq \mathbf{X}_{s}^{M} \leq \mathbf{X}_{s}^{M, U}, \mathbf{X}^{M, L} \leq \mathbf{X}^{M} \leq \mathbf{X}^{M, U}
\end{array}
$$

The equality constraint for consistency among disciplines given in the above model can be modified using $\mathbf{X}_{s}^{*,(i),(k-1)}, \mathbf{X}_{j}^{*,(i),(k-1)}, \mathbf{P}_{j}^{*,(i),(k-1)}$ into the following form:

$$
y_{j m}^{*,(i)}=y_{j m}^{*,(i)}\left(\mathbf{d}_{s}, \mathbf{d}_{j}, \mathbf{X}_{s}^{M}-\mathbf{S}_{s}, \mathbf{X}_{j}^{M}-\mathbf{S}_{j}, \mathbf{P}_{j}^{*,(i),(k-1)}, \mathbf{Y}_{\bullet j}^{*,(i)}\right) .
$$

The probability/possibility analysis model under the environment of MDO can be expressed as

$$
\begin{aligned}
& \max _{\left(\begin{array}{l}
\mathbf{U}_{\mathbf{X}, s, c}^{(i)}, \mathbf{X}_{s, d}^{(i)}, \mathbf{V}_{\mathbf{X}, s}^{(i)}, \mathbf{U}_{\mathbf{X}, c}^{(i)} \\
\mathbf{X}_{d}^{(i)}, \mathbf{V}_{\mathbf{X}}^{(i)}, \mathbf{U}_{\mathbf{p}, c}^{(i)}, \mathbf{P}_{d}^{(i)}, \mathbf{V}_{\mathbf{p}}^{(i)}
\end{array}\right)} G_{i}\left(\mathbf{d}_{s}, \mathbf{d}, \mathbf{U}_{\mathbf{X}, s, c}^{(i)}, \mathbf{X}_{s, d}^{(i)}, \mathbf{V}_{\mathbf{X}, s}^{(i)},\right. \\
& \left.\mathbf{U}_{\mathbf{X}, i, c}^{(i)}, \mathbf{X}_{i, d}^{(i)}, \mathbf{V}_{\mathbf{X}, i}^{(i)}, \mathbf{U}_{\mathbf{p}, i, c}^{(i)}, \mathbf{P}_{i, d}^{(i)}, \mathbf{V}_{\mathbf{p}, i}^{(i)}, \mathbf{Y}_{\bullet i}^{(i)}\right) \\
& \text { s.t. }\left\|\left(\mathbf{U}_{\mathbf{X}, s, c}^{(i)}, \mathbf{U}_{\mathbf{X}, c}^{(i)}, \mathbf{U}_{\mathbf{p}, c}^{(i)}\right)\right\|_{2} \leq \beta_{t} \\
& \|\left(\mathbf{V}_{\mathbf{X}, s}^{(i)}, \mathbf{V}_{\mathbf{X}}^{(i)}, \mathbf{V}_{\mathbf{p}}^{(i)} \|_{\infty}<1-\alpha_{t}\right. \\
& y_{j m}^{(i)}=y_{j m}^{(i)}\left(\mathbf{d}_{s}, \mathbf{d}_{j}, \mathbf{U}_{\mathbf{X}, s, c}^{(i)}, \mathbf{X}_{s, d}^{(i)}, \mathbf{V}_{\mathbf{X}, s}^{(i)},\right. \\
& \left.\mathbf{U}_{\mathbf{X}, j, c}^{(i)}, \mathbf{X}_{j, d}^{(i)}, \mathbf{V}_{\mathbf{X}, j}^{(i)}, \mathbf{U}_{\mathbf{p}, j, c}^{(i)}, \mathbf{P}_{j, d}^{(i)}, \mathbf{V}_{\mathbf{p}, j}^{(i)}, \mathbf{Y}_{\bullet j}^{(i)}\right) \\
& i=1,2, \cdots, n d ; j=1,2, \cdots, n d ; m=1,2, \cdots, n d ; \mathrm{j} \neq \mathrm{m}
\end{aligned}
$$

$$
\beta_{t} \text { is equal to }-\Phi^{-1}\left[\frac{\alpha_{t}}{\sum_{k=1}^{N}\left\{F_{\mathbf{X}_{r d}}\left(\mathbf{x}_{r d}^{k}\right) \times F_{\mathbf{P}_{r d}}\left(\mathbf{P}_{r d}^{k}\right)\right\}}\right] .
$$

The values of $\mathbf{d}_{s}, \mathbf{d}$ are obtained from the deterministic RFCDV-MDO. The solutions of the uncertainty analysis problem are MPPPs represented by $\left(\mathbf{U}_{\mathbf{X}, s, c}^{*,(i)}, \mathbf{X}_{s, d}^{*,(i)}, \mathbf{V}_{\mathbf{X}, s}^{*,(i)}, \mathbf{X}_{d}^{*,(i)}, \mathbf{U}_{\mathbf{X}, c}^{*,(i)}, \mathbf{V}_{\mathbf{X}}^{*,(i)}, \mathbf{U}_{\mathbf{P} c}^{*,(i)}, \mathbf{P}_{d}^{*,(i)}, \mathbf{V}_{\mathbf{P}}^{*,(i)}\right)$ $(i=1, \cdots, n d)$ and the performance measures at these MPPPs. Then the MPPPs in the X-space are obtained using Rosenblatt transformation and Eq. (3). The constraints in the deterministic MDO are modified using MPPPs when the requirements of probability/possibility constraints are not all satisfied.

To deal with the discrete-continuous optimization problem, an algorithm is developed based on the algorithms of MDOP and MDOD [19-20]. 


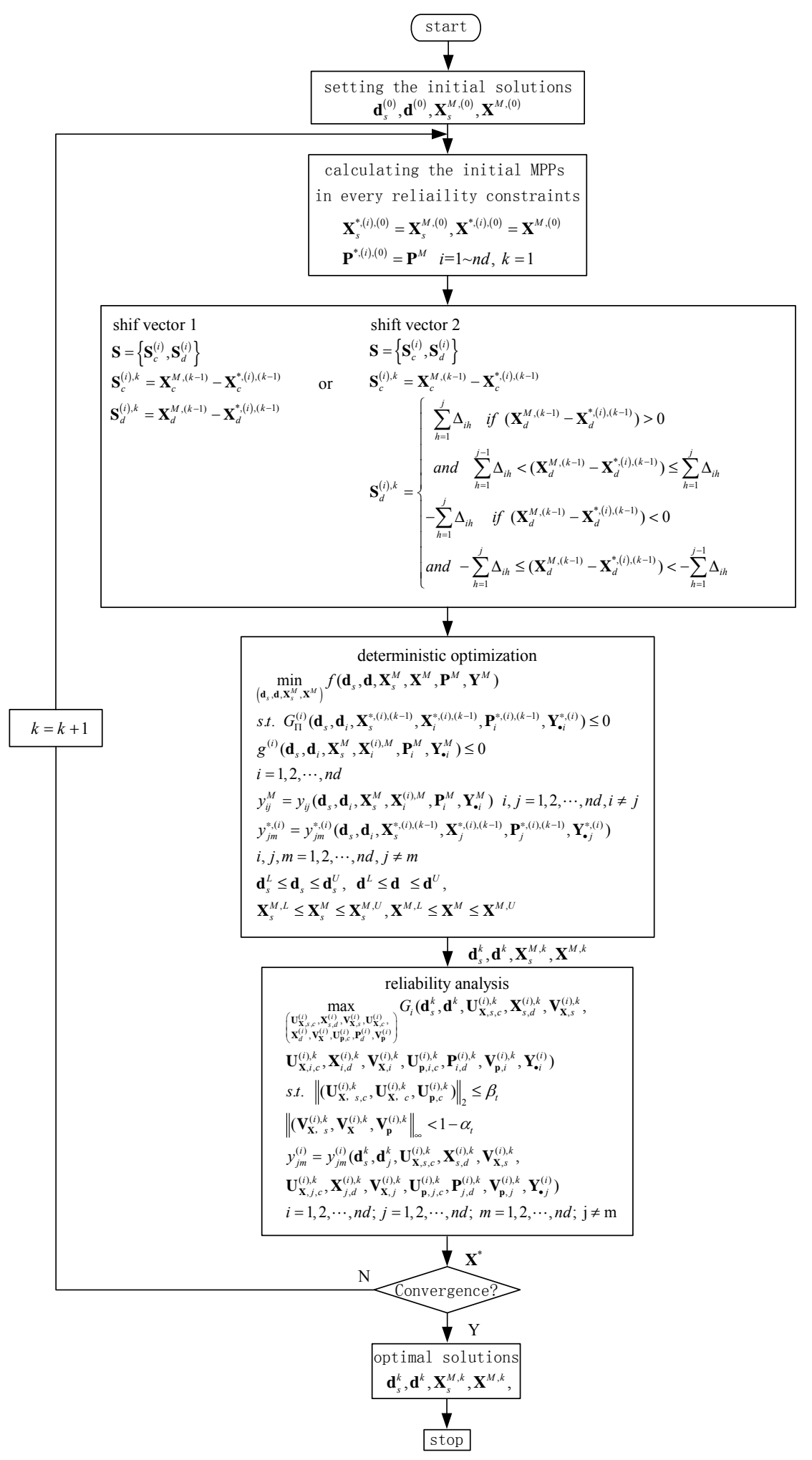

Fig. 1. Flowchart of RFCDV-MDO-SORA 


\section{Algorithm}

In the optimization formulations in Section 4, there are both inequality and equality constraints. An algorithm is developed to address the optimization problem of this type.

First, the design variables are arranged in the following sequence: $\mathbf{X}=\left[\begin{array}{ll}\mathbf{X}_{d} & \mathbf{X}_{c}\end{array}\right]$ where $\mathbf{X}_{d}=\left[x_{1}, x_{2}, \cdots, x_{m q}, \cdots, x_{m d}\right], \mathbf{X}_{c}=\left[x_{m d+1}, x_{m d+2}, \cdots, x_{m}\right]$, $x_{1}, x_{2}, \cdots, x_{m q}$ are discrete equally spaced variables; $x_{m q+1}, \cdots, x_{m d}$ are discrete and unequally spaced variables; and $x_{m d+1}, x_{m d+2}, \cdots, x_{m}$ are continuous variables.

Secondly, the equality constraints are transformed into inequality constraints. For example: if the equality constraint is $h(\mathbf{X})=0$, then the corresponding inequality constraint is $h(\mathbf{X}) \leq 0$. Replace $h(\mathbf{X})=0$ with $h(\mathbf{X}) \leq 0$ in the original optimization and modify the objective function by adding a penalty term which is equal to the square of the inequality constraint multiplied by a penalty coefficient.

\subsection{Definitions}

Definition 1: Discretization of continuous variable transforms each continuous variable into a discrete variable meeting the specified precision requirement. In this paper, the continuous variable is discretized in equal distance.

Definition 2: The hybrid discrete point is denoted by $\mathbf{X}^{D} \in \mathbf{E}^{m}=\mathbf{E}^{D} \cup \mathbf{E}^{C}$, where $\mathbf{E}^{D}$ is the value space composed of all discrete variables and $\mathbf{E}^{C}$ composed of discretized values of all continuous variables.

Definition 3: The discrete unit area of point $\mathbf{X}^{D}$, denoted by $U N\left[\mathbf{X}^{D}\right]$, is defined as

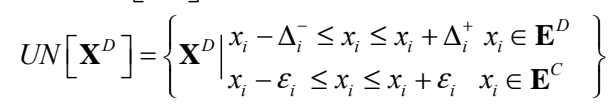

where $\Delta_{i}^{-}$and $\Delta_{i}^{+}$are discrete increments of $x_{i}$ along the negative and the positive directions, respectively. $\varepsilon_{i}$ is the distance of discretization of $x_{i}$ which is a continuous variable.

Definition 4: The discrete feasible area of constraints $\Omega$ is the aggregation of hybrid discrete points which satisfy the requirements of the constraints.
Definition 5: The local discrete optimum, $\mathbf{X}^{*}$, is a hybrid point and $\mathbf{X}^{*} \in \Omega$, among all the points $\mathbf{X} \in U N\left[\mathbf{X}^{D}\right] \cap \Omega$, we have $f\left(\mathbf{X}^{*}\right) \leq f(\mathbf{X})$.

Definition 6: The global discrete optimal point, $\mathbf{X}^{*}$, is a hybrid point and $\mathbf{X}^{*} \in \Omega$, among all the points $\mathbf{X} \in \Omega$, we have $f\left(\mathbf{X}^{*}\right) \leq f(\mathbf{X})$.

Definition 7: The matrix of discrete values. After the discretization of continuous variables, all design variables are treated as discrete variables. Suppose the number of design variables is $m$ and the maximal number of discrete values among all discrete variables is $t$. The matrix is

$$
Q=\left[\begin{array}{cccc}
q_{11} & q_{21} & \cdots & q_{m 1} \\
q_{12} & q_{22} & \cdots & q_{m 2} \\
\vdots & \vdots & \vdots & \vdots \\
q_{1 t} & q_{2 t} & \cdots & q_{m t}
\end{array}\right]
$$

where $q_{i, j-1} \leq q_{i, j} \leq q_{i, j+1}$. If the number of variables $x_{i}$ is $l$ which is less than $t$, the values of $q_{i, j}(l+1 \leq j \leq t)$ is equal to $q_{i, l}$.

Definition 8: A discrete active constraint is such a constraint such that if each component of the distance, which is from the current point to the constraint along the current search direction, is less than the increment of each variable. The increment is $\Delta_{i}^{-}$if the component of the search direction is less than zero, otherwise is $\Delta_{i}^{+}$ [19-20].

\subsection{Hybrid discrete-continuous algorithm}

The hybrid discrete-continuous algorithm is proposed in Section 5.2.1. The critical techniques used in this algorithm are interpreted in Section 5.2.2.

\subsubsection{Hybrid disrete-continuous algorithm}

Step 1: Treat all variables including discrete variables as continuous variables first; solve the optimization problem using a continuous search algorithm, for example sequential quadratic programming (SQP). The optimum point is denoted by $\mathbf{X}_{c}^{*}$. Set a vessel to save the new point.

Step 2: Discretize continuous variables, construct the matrix $Q$. Round the optimum point $\mathbf{X}_{c}^{*}$ to the discrete point $\mathbf{X}^{(k)}$.

Step 3: One-dimensional search. Starting from $\mathbf{X}^{(k)}$, calculate the sub-gradient at $\mathbf{X}^{(k)}$, find the step size for one-dimensional search. If there exists a better point $\mathbf{X}^{(k+1)}$, then do one-dimensional search again by starting 
from the new point, $k=k+1$; otherwise go to step 4 . During this process, when a new point is obtained, the point and the values of the objective function and the constraints are all saved into the vessel. When the values of the objective function and the constraints at a new point need to be calculated, the point is firstly compared with the points saved in the vessel. If they are the same point, values of the objective function and the constraints are set as the same as those saved in the vessel, otherwise the values of its objective and the constraints need to be calculated and saved in the vessel.

Step 4: Adjacent point-checking in discrete unit area of $\mathbf{X}^{(k)}$. If there exists a better point $\mathbf{X}^{(k+1)}, k=k+1$ go to step 3; otherwise go to step 5. The same strategy in Step 3 is adopted in this process.

Step 5: Fixing the discrete part of the optimum, start from the continuous part of the optimum and solve the optimization problem based on the algorithm used for the continuous optimization. Stop.

\subsubsection{Critical techniques}

(i) Round

Step 1: Directly round the optimum point $\mathbf{X}_{c}^{*}$ to the nearest discrete point $\mathbf{X}^{(k)}$.

Step 2: Check all the inequality constraints in the original optimization problem including the inequality constraints transformed from equality constraints at $\mathbf{X}^{(k)}$. If all inequality constraints are satisfied, stop; otherwise the violated constraints $g_{i}(\mathbf{X})$ are included in the set of If .

Step 3: Calculate the gradient of the constraints $g_{i}(\mathbf{X}) i=1, \cdots, n_{I f}$, where $n_{I f}$ is the number of violated constraints in the If set. The unit gradient $\mathbf{u}_{i}$ is defined as $\mathbf{u}_{i}=\frac{\nabla g_{i}(\mathbf{X})}{\left\|\nabla g_{i}(\mathbf{X})\right\|}$.

Step 4: Calculate the normalized value $\left(g_{n i}(\mathbf{X})\right)$ of constraint $g_{i}(\mathbf{X}) i=1, \cdots, n_{I f}$ :

$$
g_{n i}(\mathbf{X})=\frac{g_{i}(\mathbf{X})}{\left\|\nabla g_{i}(\mathbf{X})\right\|} \cdot w=\sum_{i}\left|g_{n i}(\mathbf{X})\right| i=1, \cdots, n_{I f} .
$$

Step 5: Calculate the weighted coefficient $\beta_{i}$ :

$$
\begin{array}{r}
\beta_{i}=\frac{w}{g_{n i}(\mathbf{X})}, \\
g_{n i}(\mathbf{X})=\left\{\begin{array}{lr}
1-\left(g_{n i}(\mathbf{X})\right)^{4} & 0.00001 \leq g_{n i}(\mathbf{X})<0.9 \\
\frac{1}{2+g_{n i}(\mathbf{X})} & g_{n i}(\mathbf{X}) \geq 0.9 . \\
1 & g_{n i}(\mathbf{X})<0.00001
\end{array}\right.
\end{array}
$$

Step 6: Calculate the feature vector $\mathbf{d w}$ : $\mathbf{d w}=\sum_{i} \beta_{i} \mathbf{u}_{i} \quad i=1, \cdots, n_{\text {If }}$, its unit vector $\mathbf{d}=\frac{\mathbf{d} \mathbf{w}}{\|\mathbf{d w}\|}$.

Step 7: Using the sign of each component of $-\mathbf{d}$ to adjust $\mathbf{X}^{(k)}$, then $\mathbf{X}^{(k+1)}$ is obtained; $k=k+1$, go to Step 2.

Step 3 to Step 6 are based on [19] with the part that is different from [19] is that the direction is used to directly round instead of the direction for searching.

(ii) Construct the sub-gradient

The sub-gradient of the objective function is defined as

$$
\nabla f=\left[\frac{\nabla f}{\Delta x_{1}}, \cdots, \frac{\nabla f}{\Delta x_{m d}}, \frac{\nabla f}{\Delta x_{m d+1}}, \cdots, \frac{\nabla f}{\Delta x_{m}}\right]^{T}
$$

For the discrete variables:

$$
\frac{\nabla f}{\Delta x_{i}}=\left\{\begin{array}{ll}
\frac{f\left(\mathbf{X}+\Delta_{i} e_{i}\right)-f(\mathbf{X})}{\Delta x_{i}} & x_{i}<x_{i}^{u p} \\
\frac{f(\mathbf{X})-f\left(\mathbf{X}-\Delta_{i} e_{i}\right)}{\Delta x_{i}} & x_{i}=x_{i}^{u p}
\end{array}, \quad i=1,2, \cdots, m d\right.
$$

where $x_{i}^{u p}$ is the upper boundary of $x_{i}, e_{i}$ is a $m$ dimensional unit vector and the $i$ th component is one.

For the continuous variables:

$$
\frac{\nabla f}{\Delta x_{i}}=\left\{\begin{array}{lc}
\frac{f\left(\mathbf{X}+\varepsilon_{i} e_{i}\right)-f(\mathbf{X})}{\varepsilon_{i}} & x_{i}<x_{i}^{u p} \\
\frac{f(\mathbf{X})-f\left(\mathbf{X}-\varepsilon_{i} e_{i}\right)}{\varepsilon_{i}} & x_{i}=x_{i}^{u p}
\end{array}, \quad i=m d+1, \cdots, m\right.
$$

The hybrid negative sub-gradient direction $\mathbf{d}$ is defined as:

$$
\mathbf{d}=\left\{d_{i}=-\frac{\nabla f / \Delta x_{i}}{D}, i=1, \cdots, m\right\} \quad \text { where } \quad D \quad \text { is }
$$
$\max \left|\frac{\nabla f}{\Delta x_{i}}\right|, \quad i=1, \cdots, m$

The sub-gradient of the constraint function is similarly defined as that of the objective function.

(iii) Step size

Suppose the current point is $\mathbf{X}^{(k)}$, then the new point is: $\mathbf{X}^{(k+1)}=\mathbf{X}^{(k)}+a_{k} \mathbf{d}^{(k)}$ 
Expanding the constraint $g_{i}(\mathbf{X})$ at $\mathbf{X}^{(k)}$ : $g_{i}\left(\mathbf{X}^{(k+1)}\right)=g_{i}\left(\mathbf{X}^{(k)}\right)+\nabla g_{i}^{T}\left(\mathbf{X}^{(k)}\right)\left(\mathbf{X}^{(k+1)}-\mathbf{X}^{(k)}\right)$

To ensure $g_{i}\left(\mathbf{X}^{(k+1)}\right) \leq 0$, we need to have $a_{k} \leq-\frac{g_{i}\left(\mathbf{X}^{(k)}\right)}{\nabla g_{i}^{T}\left(\mathbf{X}^{(k)}\right) \mathbf{d}^{(k)}}=a_{i}$, because the constraint with $\nabla g_{i}^{T}\left(\mathbf{X}^{(k)}\right) \mathbf{d}^{(k)}>0$ can prevent the decrease of the objective function. Thus, only the constraint with $\nabla g_{i}^{T}\left(\mathbf{X}^{(k)}\right) \mathbf{d}^{(k)}>0$ is considered. Set $a_{k}=\min \left\{a_{i}, a_{i} \geq 0\right\}$ [19].

(iv) One-dimensional search

Step 1: Set the search precision $\varepsilon>0$ and the reduction proportion $\gamma(0<\gamma<1)$.

Step 2: Calculate $a_{k}$.

Step 3: Calculate the new point $\mathbf{X}^{(k+1)}$ : $\mathbf{X}=\mathbf{X}^{(k)}+a_{k} \mathbf{d}^{(k)}, \mathbf{X}^{(k+1)}$ is the direct round result of $\mathbf{X}$.

Step 4: Check the feasibility of $\mathbf{X}^{(k+1)}$ : if $g_{i}\left(\mathbf{X}^{(k+1)}\right) \leq 0 \quad i=1, \cdots, n_{\text {ueq }}+n_{\text {eq }} ; f\left(\mathbf{X}^{(k+1)}\right)<f\left(\mathbf{X}^{(k)}\right)$, then go to Step 2; otherwise $a_{k}=a_{k}-\gamma a_{k}$, go to Step 3 until $a_{k} \leq \varepsilon . n_{u e q}$ and $n_{e q}$ are the numbers of inequality and equality constraints in the original problem, respectively.

\section{(v) Adjacent point-checking}

Using the one-dimensional search technique, in most cases, we get only a local optimum point. Then the adjacent point-checking technique in [19] is used. First the point-checking direction $v$ is determined as:

$$
\begin{aligned}
& v=\alpha_{1} s_{1}+\alpha_{2} s_{2} \\
& \alpha_{1}=\frac{1}{\left\|s_{1}\right\|} \quad \alpha_{2}=\frac{1}{\left\|s_{2}\right\|} \\
& s_{1}=\nabla G^{T} \nabla f \nabla G-\nabla f \\
& s_{2}=\frac{\nabla G}{\nabla G^{T} \nabla f}-\nabla f \\
& \nabla G=\frac{1}{k} \sum \nabla g_{i}
\end{aligned}
$$

where $k, \nabla g_{i}$ are the number and the sub-gradient of the discrete active constraints, respectively.

Step 1: Calculate the direction $v$.

Step 2: Do one-dimensional search along $v$.

Step 3: If

$g_{i}\left(\mathbf{X}^{(k+1)}\right) \leq 0 \quad i=1, \cdots, n_{\text {ueq }}+n_{\text {eq }} ; f\left(\mathbf{X}^{(k+1)}\right)<f\left(\mathbf{X}^{(k)}\right)$, then

stop; other go to Step 4.

Step 4: Adjacent point-checking using the method of [20]. During this process a better point is defined as
$g_{i}\left(\mathbf{X}^{(k+1)}\right) \leq 0 \quad i=1, \cdots, n_{\text {ueq }}+n_{\text {eq }} ; f\left(\mathbf{X}^{(k+1)}\right)<f\left(\mathbf{X}^{(k)}\right), n_{\text {ueq }}$ and $n_{e q}$ are the numbers of inequality and equality constraints in the original problem respectively.

\subsection{Analysis of algorithm}

The discrete one-dimensional search starts at a point which is the result of rounding at the optimum of the continuous optimization. Generally the discrete global optimal point can not be attained by the onedimensional search. The adjacent point-checking technique is conducted then among the discrete unit area to find a new and better point with the aim of escaping from the local optimum. After this process has been repeated several times, the optimum point obtained is the best point in all discrete points of the discretized problem. Finally the values of the original discrete variables are fixed at the relevant values of that optimum. A continuous optimization is carried out while starting at the continuous part of that optimum.

\section{Examples}

In this section, the proposed RFCDV-MDO model and the solution approach of the RFCDV-MDO model within the framework of Sequential Optimization and Reliability Assessment are demonstrated using a mathematical example and an engineering design example.

\subsection{Mathematical example for RFCDV-MDO}

This mathematic example is based on from the one in [14]:

$$
\begin{aligned}
& \min _{\left(d_{s}, d_{1}, d_{2}\right)} f\left(\mathbf{d}, \mathbf{x}^{M}\right)=\left(d_{s}+x_{s}^{M}\right)^{2}+d_{1}^{2}+d_{2}^{2} \\
& \text { s.t. } \quad \Pi\left\{G_{1}(\mathbf{d}, \mathbf{x})=x_{1}-d_{s}-x_{s}-d_{1}-d_{2}>0\right\} \leq \alpha_{t} \\
& \quad \Pi\left\{G_{2}(\mathbf{d}, \mathbf{x})=d_{s}+x_{s}-2 d_{1}+d_{2}-x_{2}>0\right\} \leq \alpha_{t} \\
& 0 \leq d_{s}, d_{1}, d_{2} \leq 5 \\
& d_{s}, d_{1}, d_{2} \text { are design variables and take values as } \\
& \text { multiples } \text { of } 0.01 \quad \text { in this formulation. } \\
& x_{s} \sim\left\{\begin{array}{cl}
-0.24 & p=0.2 \\
0 & p=0.6, x_{1} \sim N(5,0.5), \\
0.24 & p=0.2
\end{array}\right.
\end{aligned}
$$

where $N(\mu, \sigma)$ stands for a normal distribution with the mean value of $\mu$ and the standard deviation of $\sigma$. The triangular membership function of $x_{2}$ is denoted by 
$(0.7,1,1.3)$. In $\left(x^{M}-d t, x^{M}, x^{M}+d t\right)$, the value $x^{M}$ is the maximal grade point of membership function of $x$; the value $d t$ is the deviation on each side from the maximal grade point. The problem is decomposed into two subsystems as shown in Fig. 2 following the same approach as used in [14].

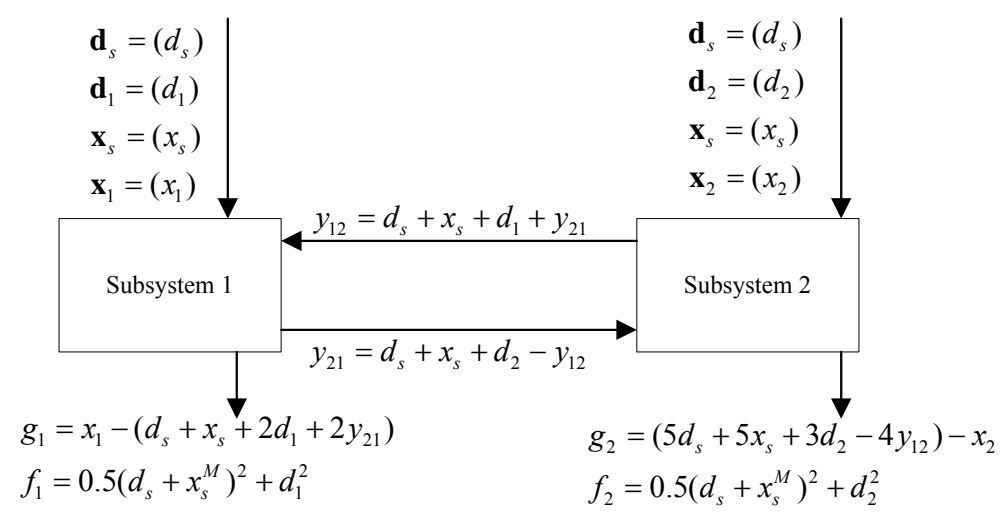

Fig. 2. The mathematical problem and its decomposition

It should be noted that, $x_{s}, x_{1}, x_{2}$ are all design parameters but not design variables in this problem. It is because the mean value or the maximal grade point is fixed.

The formulation of the deterministic MDO is:

$$
\begin{aligned}
& \min _{\left(d_{s}, d_{1}, d_{2}\right)} f=\left(f_{1}+f_{2}\right)=\left(d_{s}+x_{s}^{M}\right)^{2}+d_{1}^{2}+d_{2}^{2} \\
& \text { s.t. } \quad G_{1}=x_{1}^{*,(1)}-\left(d_{s}+x_{s}^{*,(1)}+2 d_{1}+2 y_{21}^{*,(1)}\right) \leq 0 \\
& y_{12}^{*,(1)}=d_{s}+x_{s}^{*,(1)}+d_{1}+y_{21}^{*,(1)} \\
& y_{21}^{*,(1)}=d_{s}+x_{s}^{*,(1)}+d_{2}-y_{12}^{*,(1)} \\
& G_{2}=\left(5 d_{s}+5 x_{s}^{*,(2)}+3 d_{2}-4 y_{12}^{*,(2)}\right)-x_{2}^{*,(2)} \leq 0 \\
& y_{12}^{*,(2)}=d_{s}+x_{s}^{*,(2)}+d_{1}+y_{21}^{*,(2)} \\
& y_{21}^{*,(2)}=d_{s}+x_{s}^{*,(2)}+d_{2}-y_{12}^{*,(2)} \\
& 0 \leq d_{s}, d_{1}, d_{2} \leq 5
\end{aligned}
$$

The optimal point $\left(d_{s}, d_{1}, d_{2}\right)$ is then used in the probability/possibility analysis. First, all continuous random variables/parameters need to be transformed into independent continuous normal random variables/parameters, the fuzzy continuous/discrete variables/parameters into standard fuzzy variables/parameters.

The formulation for searching for the MPPP of $G_{1}$ is given as:

$$
\begin{aligned}
& \max _{\left(x_{s}^{(1)}, u_{1}^{(1)}, v_{2}^{(1)}\right)} G_{1}=\left(x_{1}^{M}+u_{1}^{(1)} \sigma_{1}\right)-\left[d_{s}+x_{s}+2 d_{1}+2 y_{21}^{(1)}\right] \\
& \text { s.t. }\left\|u_{1}^{(1)}\right\|_{2} \leq \beta_{t} \\
& \left\|v_{2}^{(1)}\right\|_{\infty}<1-\alpha_{t} \\
& y_{12}^{(1)}=d_{s}+x_{s}+d_{1}+y_{21}^{(1)} \\
& y_{21}^{(1)}=d_{s}+x_{s}+d_{2}-y_{12}^{(1)}
\end{aligned}
$$

where $\beta_{t}$ is equal to $-\Phi^{-1}\left[\frac{\alpha_{t}}{\sum_{k=1}^{N} F_{X_{s}}\left(x_{s}^{k}\right)}\right]$. The variable $x_{s}^{(1)}$ is discrete and takes only a few allowable values.

The solution MPPP $\left(x_{s}^{*,(1)}, u_{1}^{*,(1)}, v_{2}^{*,(1)}\right)$ is then transformed into the MPPP $\left(x_{s}^{*,(1)}, x_{1}^{*,(1)}, x_{2}^{*,(1)}\right)$ in the Xspace. Similarly, the formulation for searching for the MPPP of $G_{2}$ can be derived while the solution is denoted by $\left(x_{s}^{*,(2)}, x_{1}^{*,(2)}, x_{2}^{*,(2)}\right)$. If the requirements of probability/possibility constraints are not all satisfied, the MPPP is used to reconstruct the deterministic MDO for the next iteration.

The stopping criterion is

$$
G_{i} \leq 0, i=1 \sim 2 ;|f(k)-f(k-1)| \leq 0.0001 .
$$

To carry out the proposed algorithm, the ranges of the linking variables need to be firstly calculated, and then be discretized. During the solution process, $\left\|v_{2}^{(1)}\right\|_{\infty} \leq 1-\alpha_{t}$ is used as the stopping criterion instead 
of $\left\|v_{2}^{(1)}\right\|_{\infty}<1-\alpha_{t}$. Theoretically this will result in a conservative solution.

The results of RBMDO with $x_{s} \sim N(0,0.3)$, $x_{1} \sim N(5,0.5), x_{2} \sim N(1,0.1)$, RBMDO solved by SORA, and RFCDV-MDO-SORA are shown in Table 1.

The probability coefficient $(\beta=3)$ in RBMDO is equivalent to the possibility coefficient $\left(\alpha_{t}=1-\Phi(\beta)=0.0013\right)$ in RFCDV-MDO. Columns two to five in Table 1 are the results of design variables and the objective function. Considering the discrete requirements in design variables, RFCDV-MDO-SORA delivers a relatively more conservative design. Both constraints at each optimal design point meet the requirements of probability or possibility. Columns eight to nine are iterations of the disciplines 1 and 2 . The disciplinary iterations in RFCDV-MDO-SORA and RBMDO-SORA are much less than that in RBMDO. Because of the introduction of SORA, and RFCDVMDO-SORA can efficiently solve RFCDV-MDO problems like SORA in RBMDO. The whole process converges in three iterations which is the same as SORA in RBMDO. Table 2 shows the process of the whole solution, where $n_{1}, n_{2}$ are the iterations needed in disciplines 1 and 2 respectively, $n_{3}$ is the iterations used in calculating the ranges of the link variables, $n_{4}$ is the number of iterations of disciplinary analysis needed in the proposed algorithm.

Table 1. Results of RBMDO, RBMDO-SORA and RFCDV-MDO-SORA

\begin{tabular}{|c|c|c|c|c|c|c|c|c|c|}
\hline & \multicolumn{3}{|c|}{ Design Variables } & \multicolumn{2}{|l|}{ Objective } & \multicolumn{4}{|c|}{ Iterations } \\
\hline & $d_{\mathrm{s}}$ & $d_{1}$ & $d_{2}$ & $f$ & $G_{1}$ & $G_{2}$ & $n_{1}$ & $n_{2}$ & $k$ \\
\hline $\begin{array}{l}\text { RBMDO Optimum } \\
\left(\beta_{\mathrm{t}}=3\right)\end{array}$ & 2.2500 & 2.2494 & 2.2499 & 15.1843 & 0 & -0.050212 & 186600 & 186600 & - \\
\hline $\begin{array}{l}\text { RFCDV-MDO-SORA optimum } \\
\left(\alpha_{\mathrm{t}}=0.0013\right)\end{array}$ & 2.2500 & 2.2500 & 2.2500 & 15.1875 & -0.0100 & -0.4604 & 1800 & 1800 & 3 \\
\hline
\end{tabular}

Table 2. Process of RFCDV-MDO-SORA

\begin{tabular}{ccccccccccc}
\hline Cycle & \multicolumn{3}{c}{ Design Variables } & \multicolumn{3}{c}{ Objective } & \multicolumn{4}{c}{ Iteration } \\
\cline { 2 - 10 } & $d_{s}$ & $d_{1}$ & $d_{2}$ & $f$ & $G_{1}$ & $G_{2}$ & $n_{1}$ & $n_{2}$ & $n_{3}$ & $n_{4}$ \\
\hline 1 & 1.6700 & 1.6700 & 1.6700 & 8.3667 & 1.7300 & -0.4604 & 600 & 600 & 387 & 213 \\
2 & 2.2500 & 2.2500 & 2.2500 & 15.1875 & -0.0100 & -0.4604 & 600 & 600 & 387 & 213 \\
3 & 2.2500 & 2.2500 & 2.2500 & 15.1875 & -0.0100 & -0.4604 & 600 & 600 & 387 & 213 \\
\hline
\end{tabular}

\subsection{Design of a pressure vessel}

In Fig. 3 the example of pressure vessel design shown is derived [21], in which the example is solved in a multiplayer formulation based on game theory. In Table 3, the nomenclature of this example is shown. Radius $(R)$, length $(L)$ and thickness $(T)$ are the design variables. There are two parameters, namely, internal pressure $(P)$ and the tensile strength of the material $\left(S_{t}\right)$. The objective is to maximize the internal volume, while minimizing the weight. This problem is modified to an MDO problem in this analysis.
By two design groups, and the coupled variables are thickness $(T)$, length $(L)$ and radius $(R)$, the pressure vessel is designed. The multidisciplinary system and the notation used are given in Fig. 4.

In this paper, $T, R$ are continuous random variables, and $L$ is a discrete random variable. Uncertainty descriptions of these design variables and parameters is shown in Table 4. 


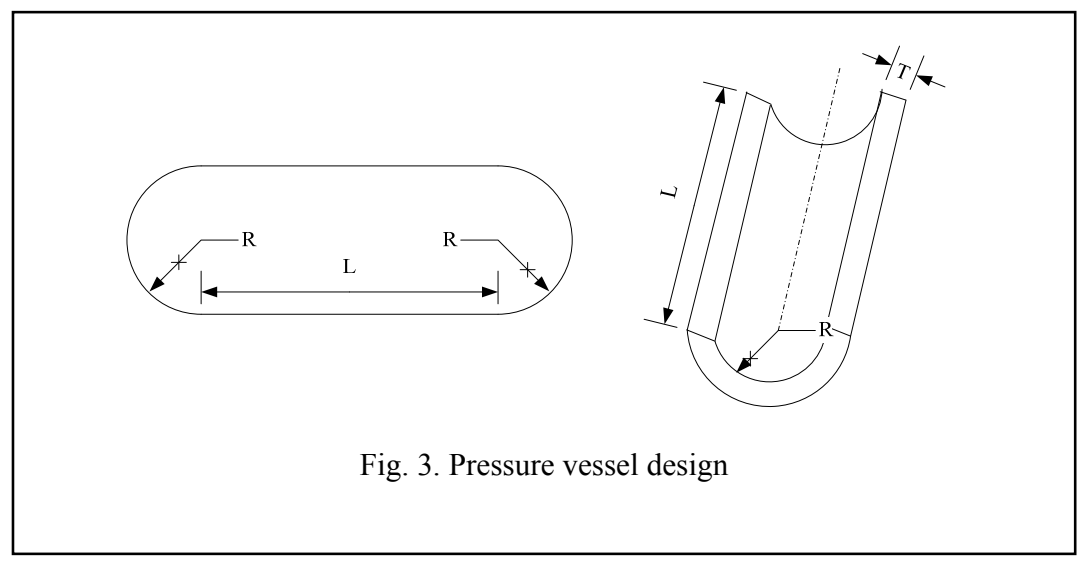

Table 3. Nomenclature of the pressure vessel design problem

\begin{tabular}{|l|l|}
\hline $\mathrm{W}$ & Weight of the pressure vessel \\
\hline $\mathrm{V}$ & Volume, in. $^{3}$ \\
\hline $\mathrm{R}$ & Radius, in. \\
\hline $\mathrm{T}$ & Thickness, in. \\
\hline $\mathrm{L}$ & Length, in. \\
\hline $\mathrm{P}$ & Internal pressure of the vessel, Klb \\
\hline $\mathrm{S}_{\mathrm{t}}$ & $\begin{array}{l}\text { Ttensile strength of the } \\
\text { cylinder material, Klb }\end{array}$ \\
\hline$\sigma_{\text {circ }}$ & Circumference stress \\
\hline
\end{tabular}

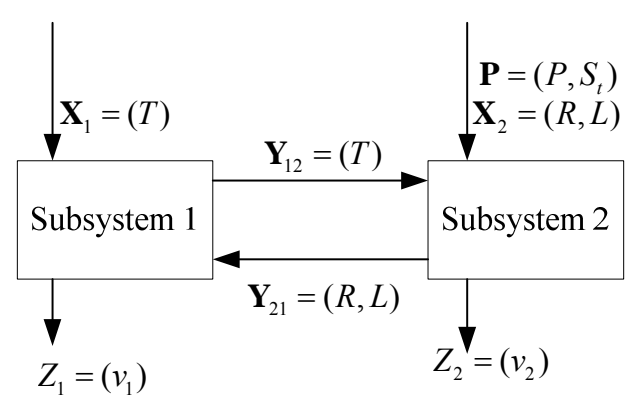

Fig. 4. System structure of the pressure vessel design problem

Table 4. Uncertainty descriptions of design variables and parameters

\begin{tabular}{|l|l|l|l|l|l|}
\hline $\begin{array}{l}\text { Variables or } \\
\text { Parameters }\end{array}$ & Mean value & Standard deviation & Distribution & $\begin{array}{l}\text { Lower boundary } \\
\text { of mean value }\end{array}$ & $\begin{array}{l}\text { Upper boundary } \\
\text { of mean value }\end{array}$ \\
\hline $\mathrm{R}$ & & 0.01 & Normal & 0.1 & 36 \\
\hline $\mathrm{T}$ & & 0.01 & Normal & 0.5 & 6.0 \\
\hline $\mathrm{L}$ & & & & 0.1 & 140 \\
\hline $\mathrm{St}$ & 40 & 4 & Normal & & \\
\hline & $\begin{array}{l}\text { Maximal grade } \\
\text { point }\end{array}$ & Deviation & $\begin{array}{l}\text { Membership } \\
\text { function }\end{array}$ & & \\
\hline $\mathrm{P}$ & 3.89 & 1.167 & Triangular & & \\
\hline
\end{tabular}


Due to manufacture errors, the mean values of $T$, $R$ are multiple of 0.01 , and that of $L$ is multiples of 0.1. The practical dimension is subjected to $N\left(T^{M}, 0.01\right)$, when the mean value of $T$ is $T^{M}$. The case of $R$ is similar to that of $T$. According to the following distribution, length $L$ is discretely distributed:

$$
\operatorname{Pr} \mid\{L=\tau\}=\left\{\begin{array}{ll}
0.1 & \tau=L^{M}+0.1 \\
0.8 & \tau=L^{M} \\
0.1 & \tau=L^{M}-0.1
\end{array}, L^{M} \text { is multiple of } 0.1\right.
$$

Sharing design variables: $\mathbf{d}_{s}=\phi$.

Sharing random/fuzzy continuous/discrete variables: $\phi$.

Subsystem 1:

Random variable: $\mathbf{X}_{1}=\{T\}$.

Input linking variables: $\mathbf{Y}_{21}=\left\{y_{21,1}, y_{21,2}\right\}=\{R, L\}$.

Output linking variables: $\mathbf{Y}_{12}=\left\{y_{12}\right\}=\{T\}$.

$$
\begin{aligned}
& Z_{1}=\left\{v_{1}\right\} \\
& \text { Output: } v_{1}=\frac{4}{3} \pi\left(T^{N}+y_{21,1}^{N}\right)^{3}+\pi\left(T^{N}+y_{21,1}^{N}\right)^{2} y_{21,2}^{N} \text {. } \\
& -\left[\frac{4}{3} \pi\left(y_{21,1}^{N}\right)^{3}+\pi\left(y_{21,1}^{N}\right)^{2} y_{21,2}^{N}\right]
\end{aligned}
$$

In this subsystem 1 , the objective is to minimize the weight which is equivalent to minimizing the relevant volume. Below are the constraints in Subsystem 1. The probability/possibility constraints are:

$G_{11}=\Pi\left\{5 T-y_{21,1}>0\right\} \leq \alpha_{t}$ $G_{12}=\Pi\left\{T+y_{21,1}-40>0\right\} \leq \alpha_{t}$

Subsystem 2:

Random variable: $\mathbf{X}_{2}=\{R, L\}$.

Input fuzzy and random parameters: $\mathbf{P}=\left\{P, S_{t}\right\}$.

Input linking variables: $\mathbf{Y}_{12}=\left\{y_{12}\right\}=\{T\}$.

Output linking variables: $\mathbf{Y}_{21}=\left\{y_{21,1}, y_{21,2}\right\}=\{R, L\}$.

Output: $Z_{2}=\left\{v_{2}\right\} \cdot v_{2}=\frac{4}{3} \pi\left(R^{N}\right)^{3}+\pi\left(R^{N}\right)^{2} L^{N}$.

The objective is to maximize internal volume in this subsystem 2. The constraints in Subsystem 2 are given below. The probability/possibility constraints are

$$
\begin{aligned}
& G_{21}=\Pi\left\{\frac{P R}{y_{12}}-S_{t}>0\right\} \leq \alpha_{t} \\
& G_{22}=\Pi\left\{L+2 R+2 y_{12}-150>0\right\} \leq \alpha_{t}
\end{aligned}
$$

The objective is to minimize $v_{1}-v_{2}$ with the possibility of failure $\alpha_{t}=0.0013$. The whole process of RFCDV-MDO-SORA with the first and second kinds of shift vector converges in three and four iterations, respectively. During the process, the starting points of the current iteration are set to be the optimal results of the previous iteration in order to improve efficiency. The probability/possibility constraint expressions at relevant MPPPs are less than zero which indicates that the requirements of all probability/possibility constraints are satisfied. The optimal results of RFCDVMDO-SORA and RBMDO-SORA are given in Table 5, and the process of RFCDV-MDO-SORA with the first or the second shift vector is given in Table 6. As shown in Table 5, the results of RFCDV-MDO-SORA are conservative compared with the results of RBMDOSORA considering the discrete design variables. As shown in Table 6 , the optimal design obtained using the second kind of shift vector is more conservative than that of the first kind. The reason is that from the second iteration, the feasible area of the reconstructed deterministic MDO with the second kind shift vector is narrower than that with the first kind. The aim of the second kind of shift vector is to avoid the situation that some equality constraints especially with the even power could not be satisfied when there are discrete requirements on design variables and shiftiness of deterministic constraints.

\section{Conclusions}

This paper proposes a formulation of RFCDV (random/fuzzy/continuous/discrete variables) Multidisciplinary Design Optimization (RFCDVMDO), a solution method of the RFCDV-MDO model within the framework of Sequential Optimization and Reliability Assessment (RFCDV-MDO-SORA), and an algorithm to deal with discrete-continuous optimization problems. 
Hong-Zhong Huang Xudong Zhang De-Biao Meng Yu Liu Yan-Feng Li

Table 5. Results of RFCDV-MDO-SORA and RBMDO-SORA

\begin{tabular}{|c|c|c|c|c|c|c|c|c|c|c|c|}
\hline & \multicolumn{3}{|c|}{ Design Variables } & \multicolumn{2}{|c|}{ Objective } & & & & & \multicolumn{2}{|c|}{ Iterations } \\
\hline & TM & $\mathrm{RM}$ & LM & V1 & $\mathrm{V} 2$ & G11 & G12 & G21 & G22 & $\mathrm{n} 1$ & $\mathrm{n} 2$ \\
\hline $\begin{array}{l}\text { RBMDO-SORA } \\
\text { Optimum }(\beta \mathrm{t}=3)\end{array}$ & 5.2475 & 34.7100 & 69.9949 & $1.7822 * 10^{5}$ & $4.4009 * 10^{5}$ & -8.3194 & $1.8666 * 10^{-11}$ & $-2.7477 * 10^{-11}$ & $8.6914 * 10^{-11}$ & 3428 & 3428 \\
\hline $\begin{array}{l}\text { RFCDV-MDO-SORA } \\
\text { Optimum with the second kind } \\
\text { of shift vector }(\alpha \mathrm{t}=0.0013)\end{array}$ & 6.0000 & 33.1600 & 71.4000 & $1.9615 * 10^{5}$ & $3.9938 * 10^{5}$ & -3.0070 & -0.7976 & -0.0592 & -0.0951 & 8375 & 8375 \\
\hline $\begin{array}{c}\text { RFCDV-MDO-SORA } \\
\text { Optimum with the first kind } \\
\text { of shift vector }(\alpha \mathrm{t}=0.0013)\end{array}$ & 6.0000 & 33.2300 & 71.3000 & $1.9658 * 10^{5}$ & $4.0105^{*} 10^{5}$ & -3.0770 & -0.4576 & $-2.0724 * 10^{-4}$ & -0.0551 & 4712 & 4712 \\
\hline
\end{tabular}

Table 6. Convergence of optimization using RFCDV-MDO-SORA with the first and second kinds of shift vector

\begin{tabular}{|c|c|c|c|c|c|c|c|c|c|c|c|c|}
\hline & cycle & $\mathrm{T}^{\mathrm{M}}$ & $\mathrm{R}^{\mathrm{M}}$ & $\mathrm{L}^{\mathrm{M}}$ & $\mathrm{V}_{1}$ & $\mathrm{~V}_{2}$ & $\mathrm{G}_{11}$ & $\mathrm{G}_{12}$ & $\mathrm{G}_{21}$ & $\mathrm{G}_{22}$ & $\mathrm{n}_{1}$ & $\mathrm{n}_{2}$ \\
\hline \multirow[t]{4}{*}{ the second kind of shift vector } & 1 & 3.5000 & 36.0000 & 71.0000 & $1.2162 * 10^{5}$ & $4.8451 * 10^{5}$ & -18.3470 & -0.4576 & 24.0076 & 0.1849 & \multirow[t]{4}{*}{8375} & \multirow[t]{4}{*}{8375} \\
\hline & 2 & 6.0000 & 33.1700 & 71.4000 & $1.9623 * 10^{5}$ & $3.9967 * 10^{5}$ & -3.0170 & -0.7876 & -0.0508 & -0.0751 & & \\
\hline & 3 & 6.0000 & 33.1600 & 71.4000 & $1.9615 * 10^{5}$ & $3.9938 * 10^{5}$ & -3.0070 & -0.7976 & -0.0592 & -0.0951 & & \\
\hline & 4 & 6.0000 & 33.1600 & 71.4000 & $1.9615 * 10^{5}$ & $3.9938 * 10^{5}$ & -3.0070 & -0.7976 & -0.0592 & -0.0951 & & \\
\hline \multirow[t]{3}{*}{ the first kind of shift vector } & 1 & 3.5000 & 36.0000 & 71.0000 & $1.2162 * 10^{5}$ & $4.8451 * 10^{5}$ & -18.3470 & -0.4576 & 24.0076 & 0.1849 & \multirow[t]{3}{*}{4712} & \multirow[t]{3}{*}{4712} \\
\hline & 2 & 6.0000 & 33.2300 & 71.3000 & $1.9658 * 10^{5}$ & $4.0105 * 10^{5}$ & -3.0770 & -0.45476 & $-2.0724 * 10^{-4}$ & -0.0551 & & \\
\hline & 3 & 6.0000 & 33.2300 & 71.3000 & $1.9658 * 10^{5}$ & $4.0105 * 10^{5}$ & -3.0770 & -0.4576 & $-2.0724 * 10^{-4}$ & -0.0551 & & \\
\hline
\end{tabular}


Two kinds of uncertainty, namely aleatory uncertainty and epistemic uncertainty, are considered in the MDO model. Because of the existence of both discrete and continuous random variables in the MDO problem, direct calculation of the probability of failure is too computationally extensive. If there are many discrete random variables and each of them may take many possible values, solving the problem becomes even more time consuming. Based on conditional possibility of failure, the possibility of failure is broadened to avoid directly solving the problem with integrals at too many combinations.

The RFCDV-MDO is decoupled into deterministic MDO and probability/possibility analysis in RFCDVMDO-SORA. The whole computational process is sequential but not nested. For RFCDV-MDO-SORA with the first kind of shift vector, the values of their mean values or maximum grade points subtracting relevant MPPPs of the previous iteration are the shifts of continuous/discrete variables. With the second kind of shift vector, the values of their means or the maximal grade points subtracting the relevant MPPPs of the previous iteration are the shifts of variables whose mean values or maximal grade points are continuous. The shifts of variables whose mean values or maximal grade points are discrete can be expanded according to the values of their means or maximal grades subtracting the relevant MPPPs of the previous iteration and discrete increments or decrements. With the examples shown, RFCDV-MDO-SORA can solve RFCDV-MDO problems efficiently in a few iterations. It seems that the RFCDV-MDO-SORA with the first kind of shift vector is more efficient than that with the second kind of shift vector. However, the reason needs to be further studied.

From the number of iterations of the disciplinary analysis in the two examples, we see that the hybrid discrete-continuous algorithm can solve the discretecontinuous optimization problem efficiently. As the consistency requirements among disciplines are added as additional design variables, the size of the problem becomes much larger than that of the original optimization problem.

Our future work will focus on developing more accurate measures to deal with these two kinds of uncertainty simultaneously in design inputs, construct more efficient frameworks to deal with RFCDV-MDO, and develop more efficient and accurate discretecontinuous optimization algorithms.

\section{Nomenclature}

$c$ : continuous design variable/parameter subscript

$d$ : discrete design variable/parameter subscript

$f$ : fuzzy design variable/parameter subscript

$g$ : common constraints

$i$ : discipline $i$ subscript

(i): discipline $i$ superscript

$k$ : $k$ th iteration superscript

$n d$ : the total number of disciplines

$r$ : random design variable/parameter subscript

$s$ : sharing design variable subscript

$v$ : standard fuzzy variables

$M$ : mean value or maximal grade point superscript

$X$ : design variable

d: the vector of deterministic design variables, which may be discrete or continuous

P: the vector of design parameters, containing random and fuzzy design parameters, which may be discrete or continuous

U: the vector of standard normal random variables/parameters in the U-space corresponding to random continuous variables/parameters in the $\mathrm{X}$-space $\mathbf{V}$ : the vector of standard fuzzy variables/parameters in the $\mathrm{V}$-space corresponding to the fuzzy continuous/continuous variables/parameters in the $\mathrm{X}$ space

$\mathbf{X}$ : the vector of uncertain design variables, containing random and fuzzy design variables, which may be discrete or continuous

$\mathbf{Y}_{. i}$ : the vector of coupling variables, input of discipline $i$ $G_{\Pi}^{(i)}$ : probability/possibility constraints of discipline $i$

$\mathbf{P}^{*,(i),(k-1)}$ : the vector of MPPP of $\mathbf{P}$ in the $i$ th discipline of the $(k-1)$ th iteration

$\mathbf{X}^{*,(i),(k-1)}$ : the vector of MPPP in the $i$ th discipline of the $(k-1)$ th iteration

$\mathbf{Y}_{\bullet i}^{*,(i)}$ : the vector of linking variables, corresponding to the probability/possibility constraint in discipline $i$; *: the most probable/possible point (MPPP) superscript $f_{\mathrm{Xrc}} / f_{\text {Prc }}$ : joint probability density function of random continuous design variables $\mathrm{X} /$ design parameters $\mathrm{P}$ $F_{\mathrm{Xrd}} / F_{\mathbf{P r d}}$ : joint cumulative distribution function of random discrete design variables $\mathrm{X} /$ design parameters $\mathrm{P}$ $\Pi_{\mathbf{X} f} / \Pi_{\mathbf{P} f}:$ membership function of fuzzy design variables $\mathrm{X} /$ design parameters $\mathrm{P}$ $\Pi[G(\mathbf{X}, \mathbf{P})>0] \leq \alpha_{t}$ : failure possibility with the failure mode defined as $G(\mathbf{X}, \mathbf{P})>0$ 
$\alpha_{t}$ : target possibility of failure

$\beta_{t}$ : target reliability index

\section{Acknowledgements}

This work is partially supported by the National Natural Science Foundation of China under contract number 51075061.

\section{References}

1. H. Z. Huang, X. Zhang, W. Yuan, D. Meng and X. Zhang, Collaborative reliability analysis under the environment of multidisciplinary design optimization. Concurrent Engineering: Research and Applications, 19(3) (2011) $245-254$.

2. H. Z. Huang, H. Yu, X. Zhang, S. Zeng and Z. Wang, Collaborative optimization with inverse reliability for multidisciplinary systems uncertainty analysis. Eng. Optim. 42(8) (2010) 763-773.

3. X. Zhang, X. L. Zhang, H. Z. Huang, Z. Wang and S. Zeng, Possibility-based multidisciplinary design optimization in the framework of sequential optimization and reliability assessment, International Journal of Innovative Computing Information and Control, 6(11) (2010) 5287-5297

4. H.Z. Huang and X. Zhang, Design optimization with discrete and continuous variables of aleatory and epistemic uncertainties, Journal of Mechanical Design, 131(3) (2009) 031006-1-031006-8.

5. X. Zhang and H.Z. Huang. Sequential optimization and reliability assessment for multidisciplinary design optimization under aleatory and epistemic uncertainties. Structural and Multidisciplinary Optimization, 40(1) (2010) 165-175.

6. X. Zhang, H.Z. Huang and H. Xu, Multidisciplinary design optimization with discrete and continuous variables of various uncertainties, Structural and Multidisciplinary Optimization, 42(4) (2010) 605-618.

7. R. H. Sues, D. R. Oakley and G. S. Rhodes, Multidisciplinary stochastic optimization, in Proc. of 10th Conf. on Engineering Mechanics (New York, 1995), pp. 934-937.

8. R. H. Sues and M. A. Cesare, An innovative framework for reliability-based MDO, in Proc. of 41st AIAA/ASME/ASCE/AHS/ASC Structures, Structural Dynamics and Materials Conf. (Atlanta, GA, 2000), pp. $1-8$.

9. P. N. Koch, B. Wujek and O. Golovidov, A multi-stage, parallel implementation of probabilistic design optimization in an MDO framework, in Proc. of 8th AIAA/NASA/ISSMO Symposium on Multidisciplinary Analysis and Optimization (Long Beach, CA, 2000).

10. D. Padmanabhan and S. Batill, Decomposition strategies for reliability based optimization in multidisciplinary system design, in Proc. of 9th AIAA/USAF/NASA/ISSMO
Symposium on Multidisciplinary Analysis and Optimization (Atlanta, GA, 2002), pp. 77-83.

11. D. Padmanabhan and S. Batill, Reliability based optimization using approximations with applications to multi-disciplinary system design, in Proc. of 40th AIAA Sciences Meeting \& Exhibit (Reno, NV, 2002).

12. X. Du and W. Chen, Concurrent subsystem uncertainty analysis in multidisciplinary design, in Proc. of 8 th AIAA/NASA/USAF/ISSMO Symposium on Multidisciplinary Analysis and Optimization (Long Beach, CA, 2000).

13. X. Du and W. Chen, Collaborative reliability analysis under the framework of multidisciplinary systems design, Optim. Eng. 6(1) (2005) 63-84.

14. X. Du, J. Guo and H. K. Beeram, Sequential optimization and reliability assessment for multidisciplinary systems design, Struct. Multidiscip. O. 35(2) (2008) 117-130.

15. H. Agarwal, J. E. Renaud and E. L. Preston, et al, Uncertainty quantification using evidence theory in multidisciplinary design optimization, Reliab. Eng. Syst. Safe. 85(1-3) (2004) 281-294.

16. W. L. Oberkampf, S. M. DeLand and B. M. Rutherford, et al, Estimation of total uncertainty in modeling and simulation, Sandia Report 2000-0824 (Albuquerque, NM, 2000).

17. B. D. Youn, K. K. Choi and L. Du, Integration of reliability and possibility-based design optimizations using performance measure approach, in Proc. of SAE World Congress (Detroit, MI, 2005).

18. L. Du and K. K. Choi, An inverse analysis method for design optimization with both statistical and fuzzy uncertainties, in Proc of ASME DETC (Philadelphia, PA, 2006).

19. J. Yu and J. Zhou, Program library of optimization methodology OPB2_principle and application (Huazhong University of Science and Technology Press, Wuhan, 1997), pp. 154-175.

20. L. Z. Chen, Optimization design methodology of discrete variables in engineering_ principle and application (China Machine Press, Beijing, 1989), pp. 135-139.

21. K. Lewis, F. Mistree, Collaborative, sequential, and isolated decisions in design, in Proc. of ASME Design Engineering Technical Conf. (Sacramento, CA, 1997). 